anales de psicología / annals of psychology

2019, vol. 35, no 2 (may), 204-213

http://dx.doi.org/10.6018/analesps.35.2.336251
(C) Copyright 2019: Editum. Servicio de Publicaciones de la Universidad de Murcia. Murcia (Spain) ISSN print edition: 0212-9728. ISSN on line edition (http://revistas.um.es/analesps): 1695-2294.

On line edition License Creative Commons 4.0: BY-NC-ND

\title{
Global functioning among middle-aged patients with chronic schizophrenia: the role of medication, working memory and verbal comprehension
} \author{
and Idoia Fernández-Marañón ${ }^{2}$ \\ 1 Faculty of Psychology, University of the Basque Country (UPV/EHU) (Spain) \\ 2 Guipuzcoa Mental Health Network. Basque Health Service-Osakidetza (Spain) \\ 3 BIODONOSTLA Health Research Institute (Spain)
}

María Ruiz-Iriondo 1,3, Karmele Salaberría 1,3, Enrique Echeburúa1,3, Álvaro Iruín 2,3, Olga Gabaldón ${ }^{2}$,

\begin{abstract}
Título: Funcionamiento global entre pacientes de mediana edad con esquizofrenia crónica: el papel de la medicación, la memoria de trabajo y la comprensión verbal.

Resumen: La fase crónica de la esquizofrenia se caracteriza por la progresión de la enfermedad y por las dificultades que presentan los pacientes para volver al nivel de funcionamiento premórbido. El objetivo de este estudio fue describir las características de una muestra de pacientes con esquizofrenia crónica, y conocer las diferencias entre los pacientes menores y mayores de 45 años. En una muestra clínica de 77 pacientes con esquizofrenia crónica, se han evaluado síntomas básicos, el rendimiento cognitivo, el funcionamiento social y la calidad de vida. Todos los participantes obtuvieron puntuaciones muy altas en los síntomas residuales, y no se encontraron diferencias en las características sociodemográficas o clínicas entre los grupos de edad. Los resultados señalan que los pacientes más jóvenes tienen mejor rendimiento cognitivo, y los mayores, mejor funcionamiento social y calidad de vida. El número de fármacos utilizados, los valores en la escala de aprendizaje verbal diferido del SCIP, los errores en WCST, los subtests de Semejanzas y Dígitos del WAIS fueron las variables que mejor pronostican el funcionamiento global de los pacientes mayores de 45 años. Por lo tanto, aumentar nuestra comprensión de las características de la fase crónica de la enfermedad y el perfil de funcionamiento en función de la edad, puede ayudarnos a diseñar estrategias de intervención para mejorar la adaptación en pacientes jóvenes y de mediana edad con esquizofrenia crónica.

Palabras clave: Esquizofrenia crónica; edad; características clínicas; evaluación; funcionamiento; adaptación a la enfermedad.
\end{abstract}

\section{Introduction}

Schizophrenia can be defined as a severe mental illness characterised by the occurrence of symptoms that affect perception, thought, affect and behaviour. Psychotic symptoms, associated with changes in dopaminergic activation, are grouped into positive or florid symptoms and negative or deficit symptoms (Carson, 2006). There are also symptoms related to worsening of cognitive function and affective symptoms, including affective lability and anxiety.

According to epidemiological research, schizophrenia is a universal illness. Prevalence rates reported range from 0.4 to $0.7 \%$ (Saha, Chant, Welham \& McGrath, 2005), while incidence, estimated to be around 15 new cases per 100.000 people, is higher in men, urban environments and immigrants (Saha et al., 2005; McGrath et al., 2004; National Institute for Health and Clinical Excellence [NICE], 2014).

* Correspondence address [Dirección para correspondencia]: María Ruiz-Iriondo. BIODONOSTIA Health Research Institute. Dr. Begiristain s/n. 20014 Donostia-San Sebastián, Gipuzkoa (Spain).

E-mail: maria.ruiz@ehu.eus

(Article received: 02-07-2018; revised: 23-11-2018; accepted: 14-02-2019)
Abstract: The chronic phase of schizophrenia is characterized by illness progression and patients encountering difficulties to return to premorbid level of functioning. The objective of this study was to describe the characteristics of a sample of patients with chronic schizophrenia, as well to assess differences between patients under and over 45 years of age. In a clinical sample of 77 chronic schizophrenia patients, we assessed basic symptoms, cognitive performance, social functioning and quality of life. All participants obtained very high scores in residual symptoms, and no differences in sociodemographic or clinical characteristics between the age groups were found. Younger patients had better cognitive performance and older patients obtained better scores for social functioning and quality of life. Number of psychotropic drugs, verbal learning delayed of SCIP, errors in WCST, Similarities and Digit Symbol Coding of WAIS were the most important variables to predict global functioning of patients over 45 years old. Increasing our understanding of differences in characteristics of the chronic phase of the illness and the profile of functioning at different ages, may help us design intervention strategies to improve adaptation in young and middle-aged patients with chronic schizophrenia.

Keywords: Chronic schizophrenia; age; clinical features; assessment; functioning; adaptation to illness.
In most cases, the onset of the illness occurs between 18 and 23 years of age. There often is a prodromal phase with basic symptoms (disturbances in perception, thought processing, language and attention), and mainly negative and affective symptoms, before the development of full schizophrenia (Fusar-Poli et al., 2013).

Historically, the course of illness has been described as chronic but it does indeed become chronic in just $40 \%$ of cases (Schultze-Lutter, Ruhrmann \& Klosterkötter, 2006). Recent studies have found that between 25 and $30 \%$ of patients achieve a complete recovery of their premorbid activity and functioning after a first episode (Klosterkötter et al., 2005; Klosterkötter, Schultze-Lutter, Benchdorf \& Rurhman, 2011; Wils et al., 2017), while the remaining patients do not achieve full remission of symptoms and will be at risk of relapse and exacerbation episodes throughout their lives.

Such relapse and exacerbation are typical of the chronic phase of the illness (Hafner \& an der Heiden, 2008), defined using the following criteria: a) duration of illness: more than five years since the first episode; b) course: patients showing various relapses; c) functional status: patients failing to achieve their premorbid functioning; and d) symptom inten- 
sity: patients having severe residual and negative symptoms (such as apathy, anhedonia, anergia, inattention and social isolation) as well as emotional lability and inability to establish social relationships. There may also be attenuated positive symptoms, such as delusional thoughts or auditory hallucinations, which are resistant to medication, and in 75 to $85 \%$ of patients, cognitive difficulties -in the form of basic symptoms- and deficits (Gray \& Roth, 2007; Bowie, Reichenberg, McClure, Leung \& Harvey, 2008).

In chronic phases, several different studies prompt that positive symptoms decrease with time while negative and depressive symptoms remain unchanged in young and middle-aged patients, and cognitive deterioration increases (Cohen et al., 2008). Longitudinal studies have found poorer scores in cognitive performance in 2.5 and 6 year follow-ups (Harvey et al, 1999, Friedman et al., 2001).

Nevertheless, few studies have specifically investigated alterations in patients with chronic schizophrenia as a function of age. Thus, the objectives of this study were to describe the psychopathological characteristics, cognitive performance, social functioning and self-perceived quality of life of patients diagnosed with chronic schizophrenia, as well as compare profiles stratifying by age into two groups: under and over 45 years. In addition, the differences in relation to the gender of participants within the different age groups have been studied.

\section{Methods}

\section{Participants}

Patients with chronic schizophrenia were recruited among users diagnosed by the psychiatrists of the Gipuzkoa Mental Health Network according to the diagnostic criteria of the $10^{\text {th }}$ revision of the International Classification of Diseases (World Health Organization, 1992). Participants were recruited by the psychiatrist among their patients' list following these inclusion criteria:

- Having $\geq 5$ years since illness onset and being between 25 and 65 years old

- Being in a stable phase of the illness and under psychopharmacological treatment

- Having negative or attenuated positive symptoms

- Failing to achieve premorbid functioning in terms of education, work and/or social life

- Having no diagnosis of mental retardation or associated neurological disorders

- Agreeing to participate in the study and giving written informed consent

115 patients were selected, and 77 agreed to participate in the study and were stratified as a function of age into two groups: under 45 years old ( 35 individuals) and 45 years old or above ( 42 individuals) because the 45 years was the median of the sample.

\section{Instruments}

All the assessment instruments used have been benchmarked in and adapted for the Spanish language and have appropriate psychometric properties.

\section{Sociodemographic data}

We carried out an interview to collect sociodemographic data such as sex, marital status, number of offspring, level of education and employment status, as well as the following data related to patients' clinical history: diagnosis, illness duration, total number of psychiatric admissions and usual pharmacological treatment (amount and type of medication).

Frankfurt Complaint Questionnaire (FCQ; Sülwood \& Huber, 1986). This is a self-report test composed of 98 items measuring the presence of the "basic" symptoms of schizophrenia. It contains 10 subscales that gather information about the symptoms that represent the substrate linking the underlying organic causes and the symptoms of the illness itself that are manifested in prodromal and residual phases. We used a validated version for the Spanish population, in which Cronbach's alpha was above .95 and the test-retest reliability was above .60 (Jimeno-Bulnes, Jimeno-Valdés \& VargasAragón, 1996).

Brief Psychiatric Rating Scale (BPRS; Overall \& Gorham, 1962; Peralta \& Cuesta, 1994). This is an intervieweradministered test with 18 items rated on a Likert-type scale ranging from 0 (lack) to 4 (very severe). This instrument assesses the presence of symptoms of anxiety, depression, thought disorders, anergia, activation and hostility-suspiciousness.

Screen for Cognitive Impairment in Psychiatry (SCIP; Pino et al., 2006; Purdon, 2005). This test assesses immediate and delayed verbal learning, working memory, verbal fluency and information processing speed. It is used for identifying cognitive impairment. The test-retest reliability ranged from .74 to .90 and Cronbach's alpha was .73 .

Wechsler Adult Intelligence Scale-Third Edition (WAIS-III; Weschler, 1999). Short forms of this scale have been designed for patients with schizophrenia. Scores on these short forms are correlated (.91) with the overall intelligence quotient (IQ) of the full scale in clinical patients (Fuentes-Durá, Romero-Peris, Dasí-Vivó \& Ruiz-Ruiz, 2010).

Wisconsin Card Sorting Test (WCST; Heaton, Chelune, Talley, Kay \& Curtiss, 1993; Heaton, Chelune, Talley, Kay \& Curtiss, 2009). This test assesses attention span, planning and execution, and hence is considered a valid measure of executive function. We used the version of the test standardised and benchmarked for the Spanish population by TEA Editions.

Social Functioning Scale (SFS; Birchwood, Smith, Cochrane, Wetton \& Copestake, 1990). We used the short version of the scale, validated in a Spanish clinical sample (Torres \& Olivares, 2005), with a Cronbach's alpha of .76.

Lancashire Quality of Life Profile (LQoLP; Oliver, Huxley, Priebe \& Kaiser, 1997; Vázquez-Barquero et al., 1997). This 
instrument assesses patient satisfaction with various aspects of their life, work, leisure time, religion, finances, living situation, safety, family relations, social relations, and health, as well as global wellbeing and self-esteem.

\section{Procedure}

All the procedures performed in this study complied with the ethical standards of the University and the Ethics Committee of the Hospital. The study was conducted in accordance with the Declaration of Helsinki (World Medical Association, 2013).

Patients who met the inclusion criteria were derived by their psychiatrists of the Public Mental Health Network. Prior to beginning the study, all participants underwent informed consent procedures, which were approved by the Ethics Committee. Participants were informed of the confidentiality associated with the study and were aware that they could decline to participate and choose not to answer questions contained in the assessment protocol.

The first author of this paper provided information about the study to the patients and who agreed to participate were assessed by a psychologist (the first author) in three individual sessions in the mental health centre.

\section{Data Analysis}

Data were analyzed using the Statistical Package for Social Sciences SPSS version 23 for Mac. For all analyses, $p$ values $<.05$ were considered significant.

In this study we conducted several analyses:

- To verify the assumptions of the statistical model, Kolmogorov-Smirnov test was used to testing the normality of distribution. Levene test was used for the study of the equality of variances; Rachas test was carried out to test the independence of the observations.

- In the study of the goodness of fit of the model it was found that the assumption of normality was not met and therefore non-parametric tests have been chosen.

- Descriptive statistics were used to characterize the sample: means and standard deviations for quantitative variables, frequencies and percentages for qualitative variables.

- The sample was stratified by age to compare different characteristics. The value chosen to segment the sample is the mean of age. Likewise, the data have been segregated according to the gender of the participants in each age group. To compare the differences between the participants according to age and gender, the Mann-Whitney $U$ test and its corresponding effect size were used in quantitative, and $X^{2}$ and Kramer's $-v$ for qualitative variables.

- To analyze the weight of various differential variables statistically associated with the variable criteria, more or less 45 years, we used logistic regression. The predictive value of the model was evaluated with the Nagelkerke $R^{2}$ and to the goodness of fit was obtained by the $X^{2}$ and the Hosmer-Lemeshow index.

\section{Results}

\section{Sociodemographic and clinical characteristics}

The main sociodemographic and clinical results are summarised in Table 1.

Table 1. Sociodemographic and clinical characteristics.

\begin{tabular}{|c|c|c|c|c|c|c|c|c|c|c|c|c|}
\hline \multirow[t]{3}{*}{ Variable } & \multicolumn{4}{|c|}{ Total $N=77$} & \multicolumn{4}{|c|}{$<45$ years old $N=35$} & \multicolumn{4}{|c|}{$\geq 45$ years old $N=42$} \\
\hline & \multicolumn{2}{|c|}{ Male } & \multicolumn{2}{|c|}{ Female } & \multicolumn{2}{|c|}{ Male } & \multicolumn{2}{|c|}{ Female } & \multicolumn{2}{|c|}{ Male } & \multicolumn{2}{|c|}{ Female } \\
\hline & $N$ & $\%$ & $N$ & $\%$ & $N$ & $\%$ & $N$ & $\%$ & $N$ & $\%$ & $N$ & $\%$ \\
\hline$\overline{\text { Sex }}$ & 53 & 68.8 & 24 & 31.2 & 24 & 68.57 & 11 & 31.42 & 29 & 65.04 & 13 & 30.95 \\
\hline \multirow[t]{2}{*}{ Age } & $M$ & $S D$ & $M$ & $S D$ & $M$ & $S D$ & $M$ & $S D$ & $M$ & $S D$ & $M$ & $S D$ \\
\hline & 43.5 & 8.56 & 44.04 & 10.18 & 35.75 & 5.95 & 34.91 & 6.09 & 49.97 & 3.49 & 51.77 & 5.18 \\
\hline Marital status & $N$ & $\%$ & $N$ & $\%$ & $N$ & $\%$ & $N$ & $\%$ & $N$ & $\%$ & $N$ & $\%$ \\
\hline Married & 0 & 0 & 2 & 8.3 & 0 & 0 & 0 & 0 & 0 & 0 & 2 & 15.4 \\
\hline Separated & 1 & 4.2 & 2 & 8.3 & 1 & 4.2 & 1 & 9.1 & 1 & 3.4 & 1 & 7.7 \\
\hline Widowed & 0 & 0 & 2 & 8.3 & 0 & 0 & 0 & 0 & 0 & 0 & 2 & 15.4 \\
\hline Single & 23 & 95.8 & 18 & 75 & 23 & 95.8 & 10 & 90.9 & 28 & 96.6 & 8 & 61.5 \\
\hline \multicolumn{13}{|l|}{ Children } \\
\hline Yes & 3 & 5.7 & 6 & 25 & 2 & 8.3 & 0 & 0 & 1 & 3.4 & 6 & 46.2 \\
\hline No & 50 & 94.3 & 18 & 75 & 22 & 91.7 & 11 & 100 & 28 & 96.6 & 7 & 53.8 \\
\hline \multicolumn{13}{|l|}{ Level of education } \\
\hline Primary & 18 & 34 & 10 & 41.7 & 9 & 37.5 & 4 & 36.4 & 9 & 31 & 6 & 46.2 \\
\hline Secondary & 9 & 17 & 4 & 16.7 & 3 & 12.5 & 3 & 27.3 & 6 & 20.7 & 1 & 7.7 \\
\hline Professional training & 18 & 34 & 9 & 37.5 & 8 & 33.3 & 4 & 36.4 & 10 & 34.5 & 5 & 38.5 \\
\hline University & 8 & 15 & 1 & 4.2 & 4 & 16.7 & 0 & 0 & 4 & 13.8 & 1 & 7.7 \\
\hline \multicolumn{13}{|l|}{ Employment status } \\
\hline In work & 10 & 18.9 & 6 & 25 & 7 & 29.2 & 6 & 54.5 & 3 & 10.3 & 0 & 0 \\
\hline Unemployed & 4 & 7.5 & 0 & 0 & 3 & 12.5 & 0 & 0 & 1 & 3.4 & 0 & 0 \\
\hline
\end{tabular}




\begin{tabular}{|c|c|c|c|c|c|c|c|c|c|c|c|c|}
\hline \multirow[t]{3}{*}{ Variable } & \multicolumn{4}{|c|}{ Total $N=77$} & \multicolumn{4}{|c|}{$<45$ years old $N=35$} & \multicolumn{4}{|c|}{$\geq 45$ years old $N=42$} \\
\hline & \multicolumn{2}{|c|}{ Male } & \multicolumn{2}{|c|}{ Female } & \multicolumn{2}{|c|}{ Male } & \multicolumn{2}{|c|}{ Female } & \multicolumn{2}{|c|}{ Male } & \multicolumn{2}{|c|}{ Female } \\
\hline & $N$ & $\%$ & $N$ & $\%$ & $N$ & $\%$ & $N$ & $\%$ & $N$ & $\%$ & $N$ & $\%$ \\
\hline Student & 1 & 1.9 & 0 & 0 & 8 & 33.3 & 0 & 0 & 0 & 0 & 0 & 0 \\
\hline Unable to work & 38 & 71.7 & 18 & 75 & 13 & 54.1 & 5 & 45.5 & 25 & 86.2 & 13 & 100 \\
\hline \multicolumn{13}{|l|}{ Subtype } \\
\hline Paranoid & 24 & 45.3 & 13 & 54.2 & 16 & 66.7 & 8 & 72.7 & 8 & 27.6 & 5 & 38.5 \\
\hline Simple & 6 & 11.3 & 3 & 12.5 & 2 & 8.3 & 1 & 9.1 & 13.8 & 4 & 2 & 15.4 \\
\hline Residual & 114 & 20.8 & 4 & 16.7 & 2 & 8.3 & 1 & 9.1 & 9 & 31 & 3 & 23.1 \\
\hline Hebephrenic & 5 & 9.4 & 1 & 4.2 & 0 & 0 & 0 & 0 & 5 & 17.2 & 1 & 7.7 \\
\hline Dual & 3 & 5.7 & 0 & 0 & 2 & 8.3 & 0 & 0 & 1 & 3.4 & 0 & 0 \\
\hline Affective & 2 & 3.8 & 2 & 8.3 & 1 & 4.2 & 1 & 9.1 & 1 & 3.4 & 1 & 7.1 \\
\hline Undifferentiated & 2 & 3.8 & 1 & 4.2 & 1 & 4.2 & 0 & 0 & 1 & 3.4 & 1 & 7.7 \\
\hline \multirow[t]{2}{*}{ Illness duration } & $M$ & $S D$ & $M$ & $S D$ & $M$ & $S D$ & $M$ & $S D$ & $M$ & $S D$ & $M$ & $S D$ \\
\hline & 19.28 & 7.86 & 14.75 & 6.13 & 14.75 & 6.13 & 13.55 & 3.95 & 23.03 & 7.18 & 21.69 & 7.40 \\
\hline \multirow[t]{2}{*}{ Number of admissions } & $M$ & $S D$ & $M$ & $S D$ & M & $S D$ & $M$ & $S D$ & $M$ & $S D$ & $M$ & $S D$ \\
\hline & 3.72 & 4.90 & 2.79 & 3.48 & 2.79 & 3.48 & 2.55 & 1.44 & 4.48 & 5.77 & 2.31 & 2.42 \\
\hline \multirow[t]{2}{*}{ Length of stay (months) } & $M$ & $S D$ & $M$ & $S D$ & $M$ & $S D$ & $M$ & $S D$ & $M$ & $S D$ & $M$ & $S D$ \\
\hline & 10.40 & 12.62 & 8.33 & 8.26 & 9.17 & 9.11 & 8.45 & 7.87 & 11.41 & 15.01 & 8.23 & 8.89 \\
\hline \multicolumn{13}{|l|}{ Psychosocial services } \\
\hline Yes & 38 & 71.7 & 20 & 83.3 & 15 & 62.5 & 10 & 90.9 & 23 & 79.3 & 10 & 76.9 \\
\hline No & 15 & 28.3 & 4 & 16.7 & 9 & 37.5 & 1 & 9.1 & 6 & 20.7 & 3 & 23.1 \\
\hline \multicolumn{13}{|l|}{ Medication } \\
\hline 1 & 10 & 18.9 & 4 & 16.2 & 4 & 16.7 & 1 & 9.1 & 6 & 20.7 & 3 & 23.1 \\
\hline $1+2$ & 25 & 47.5 & 9 & 37.5 & 11 & 45.8 & 2 & 18.2 & 14 & 48.3 & 7 & 53.8 \\
\hline $1+3$ & 8 & 15.1 & 5 & 20.8 & 2 & 8.3 & 3 & 27.3 & 6 & 20.7 & 2 & 15.4 \\
\hline $1+2+3$ & 9 & 17 & 4 & 16.7 & 6 & 25 & 3 & 27.3 & 3 & 10.3 & 1 & 7.7 \\
\hline $1+4$ & 1 & 1.9 & 2 & 8.3 & 1 & 4.2 & 2 & 18.2 & 0 & 0 & 0 & 0 \\
\hline
\end{tabular}

1: antipsychotics, 2: anxiolytics, 3: antidepressants, 4: others

The mean age of the sample was nearly 44 years, and most participants were single men. Almost three-quarters of the participants $(72.7 \%)$ were considered unable to work, this percentage being even higher in the older group $(90.5 \%),\left[\chi^{2}\right.$ (4) $=19.86 ; p=.001, v=.51]$ and only a fifth $(20 \%)$ were actively working, usually in sheltered employment.

Mean illness duration was more than 18 years, and longer in patients over 45 years of age [ $U=261 ; p>.000, r=.55$ ]. Nevertheless, there were not significant differences in psychiatric admissions between the groups, with a mean number of over 3 and a mean length of stay of about 10 months.

The mean age at onset was 26.08 years in women and 24.25 years in men, respectively. This age corresponds to the first episode of psychosis or appearance of positive symptoms. The most common diagnostic subtypes were paranoid (48\%) followed by residual (almost 20\%) schizophrenia. There were differences in the type of diagnosis as a function of age $\left[\chi^{2}(6)=15.83\right.$; $\left.p=.015, v=.45\right]$.

Concerning treatment, it mainly consisted of a pharmacological regimen composed of antipsychotics and anxiolytics $(44 \%)$, as well as regular individual visits by patients to their clinician. In addition, three-quarters of participants were attending some type of psychosocial resources (day hospital or centre or organisations providing sheltered employment). Under 45 years of age patients taking more psychiatric drugs (mean $=2.77, S D=1.24$ ) than the older patients $($ mean $=2.17, S D=0.88)$, and the difference was significant $[U=538 ; p=.033, r=.24]$.

Considering participants' gender in each age group, statis- tically significant differences were found in the number of children between men and women over 45 years of age [ $X^{2}$ (1) $=11.78 ; p=.001, v=.53]$.

\section{Symptoms}

Patients obtained scores higher than the cut-off for vulnerability in all the domains of the FCQ. In general, they perceived themselves as having many basic symptoms. Considering the subscales, scores significantly higher than the cut-offs were obtained in those related to the presence of speech disorders and communication difficulties. Considering the factors of the scale, depressivity and central cognitive disorder scores were above the mean, corresponding to the $68^{\text {th }}$ and $67^{\text {th }}$ percentiles, respectively. Table 2 reports the scores related to symptoms. Although scores of the older patients ( $\geq 45$ years) were higher, we did not find significant differences in perceived basic symptoms by age.

According to the BPRS, patients had relatively high scores for negative and low scores for positive symptoms, as would be expected in chronic phases of schizophrenia, and there were no significant differences between age groups (Table 2). Considering participants' gender, women over 45 years show fewer symptoms in BPRS total score [ $U=$ 116.50; $p=.047, r=.33$ ]. Among younger participants, women had lower scores in both the FCQ and the BPRS, but only significant differences appeared in the simple perception [ $U=76.50 ; p=.037, r=.35]$ and in the total score of the BPRS [ $U=68.50 ; p=.02, r=.38$ ] 
Table 2. Symptoms assessed with the Frankfurt Complaint Questionnaire (FCQ) and Brief Psychiatric Rating Scale (BPRS).

\begin{tabular}{|c|c|c|c|c|c|c|c|c|c|c|c|c|c|c|}
\hline \multirow[b]{3}{*}{ FCQ } & \multirow{2}{*}{\multicolumn{2}{|c|}{$\begin{array}{c}\text { Total } \\
N=77\end{array}$}} & \multicolumn{6}{|c|}{$<45$ years old } & \multicolumn{6}{|c|}{$>45$ years old } \\
\hline & & & \multicolumn{2}{|c|}{ Total $N=35$} & \multicolumn{2}{|c|}{ Male $N=24$} & \multicolumn{2}{|c|}{ Female $N=11$} & \multicolumn{2}{|c|}{ Total $N=42$} & \multicolumn{2}{|c|}{ Male $N=29$} & \multicolumn{2}{|c|}{ Female $N=13$} \\
\hline & $M$ & $S D$ & $M$ & $S D$ & $M$ & $S D$ & $M$ & $S D$ & $M$ & $S D$ & $M$ & $S D$ & $M$ & $S D$ \\
\hline Loss of control (0-8) & 3.96 & 2.20 & 3.74 & 2.22 & 3.50 & 2.26 & 4.27 & 2.14 & 4.14 & 2.19 & 4.38 & 2.16 & 3.62 & 2.25 \\
\hline Simple perception $(0-10)$ & 2.25 & 2.64 & 1.91 & 2.54 & 1.17 & 1.65 & 3.55 & 3.38 & 2.52 & 2.72 & 2.76 & 2.54 & 2.00 & 3.13 \\
\hline Complex perception $(0-10)$ & 3.51 & 2.52 & 3.31 & 2.41 & 2.92 & 2.20 & 4.18 & 2.71 & 3.67 & 2.63 & 3.79 & 2.55 & 3.38 & 2.87 \\
\hline Speech $(0-10)$ & 5.71 & 2.89 & 5.17 & 2.80 & 5.08 & 2.70 & 5.36 & 3.13 & 6.17 & 2.93 & 6.48 & 2.90 & 5.46 & 2.98 \\
\hline Cognition and thought $(0-10)$ & 5.49 & 2.90 & 5.43 & 3.11 & 5.08 & 2.98 & 6.18 & 3.40 & 5.55 & 2.77 & 5.59 & 2.83 & 5.46 & 2.72 \\
\hline Memory $(0-10)$ & 5.09 & 2.53 & 5.00 & 2.58 & 4.67 & 2.59 & 5.73 & 2.49 & 5.17 & 2.53 & 5.48 & 2.62 & 4.47 & 2.22 \\
\hline Motor behaviour (0-10) & 3.18 & 2.43 & 3.00 & 2.38 & 2.67 & 2.09 & 3.73 & 2.90 & 3.33 & 2.48 & 3.28 & 2.37 & 3.46 & 2.82 \\
\hline Loss of automatisms $(0-10)$ & 5.06 & 2.57 & 4.63 & 2.65 & 4.54 & 2.37 & 4.82 & 3.28 & 5.43 & 2.49 & 5.31 & 2.55 & 5.69 & 2.42 \\
\hline Anhedonia $(0-10)$ & 4.44 & 2.43 & 4.29 & 2.36 & 4.42 & 2.24 & 4.00 & 2.68 & 4.57 & 2.52 & 4.83 & 2.22 & 4.00 & 3.10 \\
\hline Irritability $(0-10)$ & 4.96 & 2.55 & 5.06 & 2.54 & 4.79 & 2.44 & 5.64 & 2.76 & 4.88 & 2.59 & 5.17 & 2.64 & 4.23 & 2.45 \\
\hline Total & 43.69 & 20.95 & 41.54 & 20.12 & 38.96 & 17.71 & 47.18 & 24.57 & 45.48 & 21.70 & 46.97 & 21.03 & 42.15 & 23.66 \\
\hline Factor 1: central & 11.03 & 5.73 & 10.49 & 5.60 & 9.58 & 5.50 & 12.45 & 5.53 & 11.48 & 5.89 & 11.48 & 6.01 & 11.46 & 5.86 \\
\hline Factor 2: percept & 8.56 & 6.99 & 7.91 & 6.66 & 6.58 & 5.14 & 10.82 & 8.73 & 9.10 & 7.30 & 9.66 & 6.93 & 7.85 & 8.20 \\
\hline Factor 3: depressivity & 13.79 & 5.35 & 13.23 & 5.27 & 13.38 & 4.92 & 12.91 & 6.20 & 14.26 & 5.45 & 14.69 & 5.41 & 13.31 & 5.63 \\
\hline Factor 4: overstimulation & 9.61 & 4.44 & 9.20 & 4.32 & 8.92 & 4.07 & 9.81 & 4.97 & 9.95 & 4.56 & 10.57 & 4.47 & 8.54 & 4.62 \\
\hline \multicolumn{15}{|l|}{ BPRS scale } \\
\hline Positive symptoms & 4.31 & 2.50 & 4.20 & 2.87 & 3.79 & 2.99 & 5.09 & 2.50 & 4.40 & 2.87 & 4.48 & 2.29 & 4.23 & 1.96 \\
\hline Negative symptoms $(0-16)$ & 8.31 & 2.12 & 8.31 & 2.03 & 8.29 & 2.33 & 8.36 & 1.50 & 8.31 & 2.18 & 8.83 & 1.83 & 7.15 & 2.51 \\
\hline Total $(0-43)$ & 22.09 & 5.84 & 21.54 & 5.91 & 20.08 & 6.38 & 24.73 & 2.93 & 22.55 & 5.82 & 23.31 & 6.04 & 20.85 & 5.11 \\
\hline
\end{tabular}

\section{Cognitive functioning}

Assessing cognitive deterioration using the SCIP, the scores in our sample were below the cut-offs proposed by Gómez-Benito et al. (2013). Participants performed worse than the scale's standardization sample. As would be expected, patients in the older group ( $\geq 45$ years) had a poorer cognitive performance, though the magnitude of the differences was small (Table 3).

Table 3. Cognitive functioning.

\begin{tabular}{|c|c|c|c|c|c|c|c|c|c|c|c|c|c|c|c|c|c|}
\hline & \multirow{2}{*}{\multicolumn{2}{|c|}{$\begin{array}{c}\text { Total } \\
N=77\end{array}$}} & \multirow{2}{*}{\multicolumn{2}{|c|}{$\begin{array}{c}<45 \text { years old } \\
N=35\end{array}$}} & \multirow{2}{*}{\multicolumn{2}{|c|}{$\begin{array}{l}\geq 45 \text { years old } \\
N=42\end{array}$}} & \multirow[b]{3}{*}{$U$} & \multirow[b]{3}{*}{$p$} & \multirow[b]{3}{*}{$r$} & \multicolumn{4}{|c|}{$\begin{array}{c}<45 \text { years old } \\
N=35\end{array}$} & \multicolumn{4}{|c|}{$\begin{array}{c}\geq 45 \text { years old } \\
N=42\end{array}$} \\
\hline & & & & & & & & & & \multicolumn{2}{|c|}{$\begin{array}{c}\text { Male } \\
N=24\end{array}$} & \multicolumn{2}{|c|}{$\begin{array}{l}\text { Female } \\
N=11\end{array}$} & \multicolumn{2}{|c|}{$\begin{array}{c}\text { Male } \\
N=29\end{array}$} & \multicolumn{2}{|c|}{$\begin{array}{l}\text { Female } \\
N=13\end{array}$} \\
\hline & $M$ & $S D$ & $M$ & $S D$ & $M$ & $S D$ & & & & $M$ & $S D$ & $M$ & $S D$ & $M$ & $S D$ & $M$ & $S D$ \\
\hline \multicolumn{18}{|l|}{$\overline{\text { SCIP }}$} \\
\hline VLT-I (cut-off < 21) & 14.78 & 4.75 & 15.71 & 4.87 & 14.00 & 4.56 & & & & 15.42 & 4.99 & 16.36 & 4.78 & 14.03 & 4.65 & 13.92 & 4.53 \\
\hline WMT (cut-off < 20) & 16.08 & 5.21 & 17.20 & 5.07 & 15.14 & 5.21 & 531 & .036 & & 17.46 & 4.61 & 16.64 & 6.15 & 15.93 & 5.43 & 13.38 & 4.35 \\
\hline VFT (cut-off < 19) & 14.55 & 4.56 & 14.51 & 4.52 & 14.57 & 4.65 & & & & 14.75 & 4.84 & 14.00 & 3.87 & 14.86 & 4.78 & 13.92 & 4.46 \\
\hline VLT-D (cut-off < 7) & 2.60 & 2.19 & 3.54 & 2.16 & 1.81 & 1.92 & 409 & .001 & .39 & 3.46 & 2.26 & 3.73 & 2.00 & 1.79 & 1.78 & 1.85 & 2.26 \\
\hline PST (cut-off $<12$ ) & 6.96 & 2.46 & 7.51 & 2.45 & 6.50 & 2.41 & 520 & .026 & .25 & 7.46 & 2.72 & 7.64 & 1.85 & 6.69 & 2.30 & 6.08 & 2.69 \\
\hline Overall score (cut-off $<70$ ) & 54.73 & 14.14 & 58.26 & 13.17 & 51.79 & 14.39 & 514 & .002 & .26 & 58.461 & 14.41 & 87.82 & 10.57 & 52.97 & 14.94 & 49.15 & 13.27 \\
\hline \multicolumn{18}{|l|}{ WAIS-III } \\
\hline Similarities & 9.18 & 2.69 & 8.49 & 3.13 & 9.76 & 2.14 & & & & 8.42 & 3.46 & 8.64 & 2.38 & 9.48 & 1.97 & 10.38 & 2.43 \\
\hline Arithmetic & 7.49 & 2.51 & 7.66 & 2.62 & 7.36 & 2.44 & & & & 8.33 & 2.84 & 6.18 & 1.17 & 7.41 & 2.32 & 7.23 & 2.77 \\
\hline Digit Span & 8.40 & 2.70 & 8.37 & 2.18 & 8.43 & 3.10 & & & & 8.50 & 2.04 & 8.09 & 2.54 & 8.48 & 3.41 & 8.31 & 2.35 \\
\hline Information & 9.08 & 2.70 & 8.34 & 3.18 & 9.69 & 2.08 & 517 & .025 & .25 & 8.83 & 3.47 & 7.27 & 2.20 & 9.86 & 1.76 & 9.31 & 2.68 \\
\hline Picture Completion & 8.66 & 2.55 & 9.11 & 2.85 & 8.29 & 2.23 & & & & 8.92 & 3.27 & 9.55 & 1.70 & 8.72 & 2.28 & 7.31 & 1.84 \\
\hline Block Design & 7.87 & 2.23 & 7.77 & 2.59 & 7.95 & 1.92 & & & & 8.00 & 2.77 & 7.27 & 2.19 & 8.10 & 1.85 & 7.62 & 2.10 \\
\hline Digit Symbol - Coding & 5.87 & 2.14 & 4.94 & 2.19 & 6.64 & 1.80 & 450 & .003 & .34 & 5.08 & 2.26 & 4.64 & 2.11 & 6.79 & 1.59 & 6.31 & 2.21 \\
\hline Full Scale IQ & 84.49 & 11.66 & 82.77 & 12.04 & 85.93 & 11.28 & & & & 83.171 & 13.53 & 81.91 & 8.38 & 86.52 & 11.89 & 84.62 & 10.09 \\
\hline \multicolumn{18}{|l|}{ WCST } \\
\hline No. of trials $(0-128)$ & 117.19 & 16.24 & 111.89 & 19.22 & 121.62 & 11.77 & 508 & .008 & .30 & 108.02 & 20.87 & 120.36 & 11.79 & 121.21 & 12.461 & 122.54 & +10.49 \\
\hline Categories completed (0-6) & 4.34 & 1.86 & 5.20 & 1.18 & 3.62 & 2.02 & 398 & .000 & .41 & 5.17 & 1.30 & 5.27 & 0.90 & 2.12 & 3.54 & 3.54 & 1.85 \\
\hline No. of correct answers (34-98) & 73.95 & 13.08 & 78.54 & 9.78 & 70.12 & 14.32 & 476.5 & .008 & .30 & 75.67 & 9.50 & 84.82 & 7.40 & 14.16 & 67.62 & 67.62 & 14.91 \\
\hline Total number of errors (10-94) & 43.12 & 20.48 & 33.34 & 15.24 & 51.26 & 20.87 & 353.5 & .000 & .30 & 2.381 & 16.40 & 35.45 & 12.79 & 20.89 & 54.77 & 54.77 & 21.22 \\
\hline No. of perseverative errors $(1-58)$ & 17.68 & 12.33 & 12.00 & 8.91 & 22.40 & 12.88 & 377.5 & .000 & .42 & 11.79 & 9.40 & 12.45 & 8.15 & 13.18 & 25.46 & 25.46 & 12.10 \\
\hline
\end{tabular}

Evaluating cognitive performance with the WAIS-III, participants obtained close to average scores in all subtests, except for the processing speed task (Digit Symbol-Coding), in which they obtained very low scores. On the other hand, the subtests in which they obtained the best scores were those related to verbal comprehension. For Similarities, Information and Digit Symbol-Coding, there were small differ- 
ences as a function of age with the older participants $(\geq 45$ years) obtaining better scores (Table 3).

In the WCST, participants needed many trials to complete the test, and though they completed many categories and made many perseverative errors. According to the cutoffs for interpreting the test scores (Heaton et al., 2009), patients had difficulties in tasks that require cognitive flexibility.

There were moderate differences in execution as a function of age in all the measures, younger patients being able to complete more categories, with fewer trials, more correct answers and fewer errors, indicating that they had less difficulty carrying out tasks related to executive function (Table 3). Considering gender, among older patients, there are no differences in cognitive functioning. Among younger participants, there are differences related to gender in the WAIS-III arithmetic test $[U=66.00 ; p=.01 ; r=.41]$ and in the number of total responses of the WCST [ $U=68.50 ; p=.02$, $r=.38]$.

\section{Social functioning and quality of life}

Social functioning scores were above the cut-offs for the scale, except in the case of prosocial behaviour, in which par- ticipants indicated difficulties in socializing (going to museums, to a cinema, or shopping in a supermarket), as well as in establishing relations in places with lots of people or noise. In this case, the younger participants $(<45$ years $)$ had more difficulties. [ $U=530.50 ; p=.03, r=.24]$

Finally, in general, patients had higher scores in selfperceived quality of life than the mean in the Spanish benchmarking study (mean $=4.67 ; S D=0.76$ ), but the older participants ( $\geq 45$ years) were more satisfied with their overall quality of life, though the difference was small [ $U=$ 531.50; $p=.028, r=.25]$.

Considering gender, younger women have better social functioning and quality of life than men. We found statistically significant differences in the execution [ $U=44.00$; $p=$ $0.001, r=.66]$ and skills items of SFS [ $U=82.50 ; p=.022, r$ $=.38]$, and living situation of LQoLP $[U=70.50 ; p=.02, r$ $=.36]$. Among older participants, women have better social functioning in execution $[U=80.50 ; p=.002 ; r=.47]$ and total score items of SFS [ $U=92.50 ; p=.009, r=.40]$, but men have a better perception of their financial situation of the LQoLP [ $U=113.50 ; p=.038, r=.32$ ]

Table 4. Social Functioning Scale and Lancashire Quality of Life Profile (LQoLP).

\begin{tabular}{|c|c|c|c|c|c|c|c|c|c|c|c|c|c|c|}
\hline & \multirow{2}{*}{\multicolumn{2}{|c|}{$\begin{array}{c}\text { Total } \\
N=77\end{array}$}} & \multirow{2}{*}{\multicolumn{2}{|c|}{$\begin{array}{c}<45 \text { years } \\
N=32\end{array}$}} & \multirow{2}{*}{\multicolumn{2}{|c|}{$\begin{array}{c}>45 \text { years } \\
N=42\end{array}$}} & \multicolumn{4}{|c|}{$<45$ years $N=32$} & \multicolumn{4}{|c|}{$>45$ years $N=42$} \\
\hline & & & & & & & \multicolumn{2}{|c|}{ Male $N=24$} & \multicolumn{2}{|c|}{ Female $N=11$} & \multicolumn{2}{|l|}{ Male $N=29$} & \multicolumn{2}{|c|}{ Female $N=13$} \\
\hline & $M$ & $S D$ & $M$ & $S D$ & $M$ & $S D$ & $M$ & $S D$ & $M$ & $S D$ & $M$ & $S D$ & $M$ & $S D$ \\
\hline \multicolumn{15}{|l|}{ Social Functioning Scale } \\
\hline Isolation-Involvement (0-6) & 4.04 & 0.99 & 3.94 & 1.11 & 4.12 & 0.88 & 3.92 & 1.21 & 4.00 & 0.89 & 4.07 & 0.92 & 4.23 & 0.83 \\
\hline Communication (0-3) & 1.77 & 0.84 & 1.66 & 0.91 & 1.86 & 0.78 & 1.63 & 0.82 & 1.73 & 1.10 & 1.90 & 0.72 & 1.77 & 0.92 \\
\hline Prosocial behaviour ( $0-12)$ & 3.91 & 2.65 & 3.17 & 2.69 & 4.52 & 2.48 & 3.29 & 2.76 & 2.91 & 2.66 & 4.52 & 2.69 & 4.54 & 2.02 \\
\hline Execution (0-9) & 6.60 & 2.25 & 6.54 & 1.90 & 6.64 & 2.53 & 5.92 & 1.82 & 7.91 & 1.30 & 5.86 & 2.64 & 8.38 & 0.96 \\
\hline Skills (0-6) & 5.52 & 0.99 & 5.49 & 1.04 & 5.55 & 0.96 & 5.25 & 1.82 & 7.91 & 1.30 & 5.38 & 1.11 & 5.92 & 0.28 \\
\hline Activities (0-3) & 0.87 & 0.99 & 0.80 & 1.02 & 0.92 & 0.97 & 1.00 & 1.10 & 0.36 & 6.00 & 0.00 & 0.79 & 0.90 & 1.23 \\
\hline Work (2-4) & 3.66 & 0.47 & 3.63 & 0.49 & 3.69 & 0.47 & 3.54 & 0.51 & 3.82 & 0.40 & 3.69 & 0.47 & 3.70 & 0.48 \\
\hline Overall scor & 26.43 & 4.95 & 25.14 & 5.00 & 27.14 & 4.79 & 24.46 & 5.48 & 26.64 & 3.50 & 25.97 & 4.87 & 29.77 & 3.49 \\
\hline \multicolumn{15}{|l|}{ LQoLP } \\
\hline Wellbeing $(1-7)$ & 4.77 & 1.19 & 4.43 & 1.19 & 5.05 & 1.12 & 4.29 & 1.30 & 4.73 & 0.90 & 5.14 & 1.02 & 4.85 & 1.34 \\
\hline Work (3-21) & 11.95 & 3.88 & 11.00 & 4.12 & 12.74 & 3.51 & 10.42 & 4.49 & 12.27 & 2.97 & 12.93 & 3.56 & 12.31 & 3.54 \\
\hline Leisure (3-21) & 14.73 & 3.23 & 14.74 & 3.21 & 14.71 & 3.28 & 14.42 & 3.14 & 15.45 & 3.39 & 14.90 & 3.33 & 14.31 & 3.27 \\
\hline Religion (2-14) & 9.65 & 12.41 & 7.63 & 2.96 & 8.71 & 2.84 & 7.29 & 2.76 & 8.36 & 3.38 & 8.41 & 3.04 & 9.38 & 2.33 \\
\hline Finances $(2-14)$ & 9.32 & 3.22 & 8.23 & 3.49 & 10.00 & 2.62 & 8.50 & 3.56 & 7.64 & 3.41 & 10.45 & 2.70 & 9.00 & 2.20 \\
\hline Living situation (7-49) & 33.32 & 6.68 & 31.91 & 7.26 & 34.50 & 5.99 & 30.42 & 6.29 & 35.18 & 8.42 & 35.21 & 5.54 & 32.92 & 6.87 \\
\hline Safety (2-14) & 10.27 & 2.60 & 9.91 & 2.83 & 10.57 & 2.39 & 9.54 & 2.78 & 10.73 & 2.87 & 11.00 & 1.95 & 9.62 & 3.04 \\
\hline Family relations (3-21) & 10.81 & 3.32 & 10.26 & 2.94 & 11.26 & 3.58 & 9.75 & 2.74 & 11.36 & 3.20 & 10.66 & 3.01 & 12.62 & 4.44 \\
\hline Social relations $(3-14)$ & 9.70 & 2.63 & 9.69 & 2.47 & 9.67 & 2.70 & 9.42 & 2.41 & 10.27 & 2.61 & 9.59 & 2.54 & 9.85 & 3.13 \\
\hline Health (3-21) & 14.96 & 3.17 & 14.74 & 3.69 & 15.14 & 2.69 & 13.96 & 3.53 & 16.45 & 3.61 & 15.14 & 2.37 & 15.15 & 3.41 \\
\hline Overall score $(1-7)$ & 5.16 & 1.32 & 4.86 & 1.57 & 5.40 & 1.04 & 4.63 & 1.74 & 5.36 & 1.75 & 5.45 & 0.91 & 5.31 & 1.32 \\
\hline
\end{tabular}

To analyze the weight of various differential variables statistically associated with the variable criteria (more or less than 45 years), we used logistic regression. The obtained model consists of number of drugs, verbal learning delayed of SCIP, errors in WCST, similarities and digit symbol coding of WAIS and shows a $X^{2}(5, N 77)=55.70 ; p<.000$ and the test of Hosmer-Lemeshow is not significant $X^{2}(8, N$ $77)=3.25 ; p<.92$. The model explains the $68.8 \%$ of the variance (Nagelkerke $\mathrm{R}^{2}$ ) and correctly classifies $80.5 \%$ of the subjects, with a level $77.1 \%$ of specificity and $83.3 \%$ of sensitivity (Table 5). 
Table 5. Predictive variables in patients $>45$ years old with chronic schizophrenia.

\begin{tabular}{|c|c|c|c|c|c|c|}
\hline Variables & $\boldsymbol{B}$ & SE & $O R$ & $95 \% \mathrm{CI}$ & Wald & $p$ \\
\hline Number of psychotropic drugs & -1.24 & 0.42 & 0.29 & {$[0.12-0.66]$} & 8.51 & .004 \\
\hline Verbal learning delayed-SCIP & -0.57 & 0.21 & 0.56 & {$[0.37-0.85]$} & 7.17 & .007 \\
\hline Total errors-WCST & 0.07 & 0.02 & 1.07 & {$[1.03-1.12]$} & 11.98 & .001 \\
\hline Similarities-WAIS & 0.39 & 0.14 & 1.48 & {$[1.11-1.97]$} & 7.31 & .007 \\
\hline Digital symbol coding-WAIS & 0.72 & 0.25 & 2.06 & {$[1.26-3.37]$} & 8.31 & .004 \\
\hline Constant & -6.39 & 2.04 & 0.00 & & 9.78 & .002 \\
\hline
\end{tabular}

\section{Discussion and conclusions}

In this study, we describe the sociodemographic and symptom profile, cognitive performance, social functioning and satisfaction with quality of life in a sample of patients with chronic schizophrenia, representative of the population seen in public health service. We also indicate differences between schizophrenia patients younger and older than 45 years of age and between men and women in each subgroup.

Awareness of such differential characteristics may help to improve the design of intervention strategies focused on the most pressing needs of patients, as well as facilitating the development of interventions focusing on the deficits of young and middle-age people with chronic schizophrenia (Mueller, Schmidt \& Roder, 2013).

Schizophrenia interrupts patients' daily lives. This reality is reflected in $36 \%$ of our sample having only completed primary education, $89 \%$ being single, and $77 \%$ being unable to work. The illness is diagnosed in people between 20 and 25 years of age, in the prime of their lives, and has a marked impact on their quality of life, expectations and goals. From the diagnostic point of view, paranoid (48\%), residual (19\%) and simple $(11 \%)$ schizophrenia were found to be the most common subtypes of schizophrenia, in line with the findings of Rocca et al. (2016). Further, patients had had a mean of three psychiatric admissions, lasting almost a year.

Regarding symptoms, participants self-report having many basic psychotic symptoms. In fact, FCQ scores were above the mean (around the $67^{\text {th }}$ percentile) in all the subscales and factors, independent of age. This is in agreement with previous research (Gross \& Huber, 1996; ShultzeLutter, 2009): basic symptoms are a core part of the illness, primary in nature and closely linked to the neurobiological substrate. Patients have anomalous subjective experiences, distinct from psychotic symptoms, that lead to secondary responses used as coping strategies, and these may occur much earlier than the manifestation of positive symptoms, as well as at the start of relapses, together with stress, and chronically as a pure defect syndrome (Comparelli et al., 2014). In this context, it seems important to study these symptoms since they persist over time, independently of age, as has been shown in a recent study (Miret, Fatjó-Vilas, Peralta \& Fañanás, 2016).

Concerning positive and negative symptoms, as would be expected in a sample of patients with chronic schizophrenia, negative symptoms were more common, and no differences being found by age ( $<$ or $\geq 45$ years). Therefore, it seems that the treatments administered in mental health services, both pharmacological and psychological, do not impact significantly on negative symptoms (Kirpatrick, Fenton, Carpenter \& Marder, 2006). Specifically, asociality, anhedonia, affective flattening and alogia are challenging to treat and represent an important gap in our understanding of the illness, given their chronic nature, their influence on patients' lives and the lack of improvement observed (Harvey \& Strassing, 2012). In recent years, patients have started to receive behavioural activation interventions, with promising results (Fernandez-Martín, Fonbellida \& Montero-Fernández, 2014).

From the cognitive point of view, our results suggest that participants show a high level of cognitive deterioration. They present poor performance in tasks associated with motor coordination, visual perception, perceptual speed, and accuracy. Also, low scores in tasks measuring processing speed are related to difficulties in visuomotor skills, distraction, and slowness, as well as lack of interest in performing the task. A review by Dickinson, Ramsey and Gold (2007) suggests that poor performance in processing speed is a key feature of cognitive impairment associated with schizophrenia. Additionally, Knowles, David and Reichenberg (2010) found that processing speed deterioration associated with schizophrenia is notably influenced by other factors, especially amount of doses of antipsychotic medication.

Regarding executive function, participants in our sample did not show severe problems of rigidity in cognitive conceptualisation and flexibility tasks, although older patients achieved poorer scores in all the sections of the WCST. Further, our patients completed more categories and made fewer perseverative errors than the participants in the study made by Bozikas, Kosmidis, Kiosseoglou and Karavatos (2006) that assessed patients of a similar type but with shorter illness duration. Our findings also differ from those of Carceller et al. (2014), whose patients, also with a shorter duration of illness, performed worse on the WCST.

Orellana and Slachevsky (2013) found that both patients, with first episodes and those with chronic schizophrenia, have difficulty inhibiting previously learned responses and fail to pay attention to relevant stimuli, and hence, they make perseverative errors. This suggests that patients present difficulties in the inhibition of inappropriate responses (Orellana \& Slachevsky, 2013)

With regards to cognitive deterioration as a function of age, several studies have found poorer performance in older patients (Bowie et al., 2008; Harvey et al., 1999; Friedman et al., 2001), above all on information processing (Granholm, 
Morris, Asarnov, Chock \& Jeste, 2000), executive function, and working memory tasks, with results being similar on other tasks, such in verbal skills (Bowie et al., 2008; Fucetola et al., 2000). Our results are in agreement with these previous findings.

In our sample, the social functioning scores were above means for the scale. Generally, participants rated their social functioning as good, especially those $\geq 45$ years of age. This finding could be explained by the fact that three-quarters of participants attended some type of psychosocial resources. Notably, however, younger individuals had lower rates of satisfaction and wellbeing in terms of socialization, and particularly in prosocial behaviour.

There may be various reasons for this: a) they still have high expectations of life that they now know they will not achieve, and this may lead to feelings of low self-efficacy; b) the burden of social-stigma and self-stigma heighten their isolation as they anticipate experiencing social rejection; and c) the low scores obtained represent the expression of the experiential dimension or lack of involvement with their environment, associated with their negative symptoms. In contrast, older patients may have adapted to their illness, having lived with it for many years (Angermeyer, Beck, Dietrich \& Holzinger, 2004; Corrigan \& Watson, 2002; García-Portilla \& Bobes, 2013; Vauth, Kleim, Wirtz \& Corrigan, 2007).

Finally, patients consider themselves to have a good quality of life. These values are also above the cut-offs for the instrument used, especially in older patients. In a previous study assessing perceived quality of life from a multiinformant perspective (Gabaldon, Ruiz-Iriondo \& Bobowik, 2013), considering ratings of the patient, as well as their assigned clinician and relatives, it was found that patients' selfassessments were the most positive. This perception may have an adaptive function, regarding the chronicity of illness and its associated limitations (Jobe \& Harrow, 2005; Fervaha, Foussias, Agid \& Remington, 2014).

To conclude, the patients assessed in our study had characteristics of chronic schizophrenia in terms of duration of illness, symptoms and cognitive performance. The basic symptoms, together with the cognitive impairment associated

\section{References}

Andreasen, R., Oades, L. G., \& Caputi, P. (2011). Conceptualizing Recovery: A consumer-oriented approach in psychological recovery: beyond mental illness. Chichester, UK: John Wiley \& Sons, Ltd. http://doi.org/10.1002/9781119975182.ch.2

Angermeyer, M. C., Beck, M., Dietrich, S., \& Holzinger, A. (2004). The stigma of mental illness: patients' anticipations and experiences. International Journal of Social Psychiatry, 50, 153-162. https://doi.org/10.1177/0020764004043115.

Birchwood, M., Smith, J., Cochrane, R., Wetton, S., \& Copestake S. (1990). The social functioning scale: the development and validation of a new scale of social adjustment for use in family intervention programmes with schizophrenic patients. British Journal of Psychiatry, 157, 853-859.

Bowie, C. R., Reichenberg, A., McClure, M. M., Leung, W. L., \& Harvey, P. D. (2008). Age- associated differences in cognitive performance in older community welling schizophrenia patients: Differential sensitivity of clinical neuropsychological and experimental information processing with chronic schizophrenia, have a negative impact on patients' functioning (Fervaha et al., 2014; Fossias et al., 2011), with a low level of prosocial behavior and a high rate, $72 \%$ of our sample, being unable to work.

\section{Clinical implications}

The implementation of multicomponent psychological treatment programs, who work on cognitive and social skills, is of interest to improve the overall functioning of patients with chronic schizophrenia and to avoid the cognitive deterioration associated with the disorder. That is, it would be useful to incorporate programs to improve cognitive functions (i.e. working memory, abstract reasoning, and executive functions), and a readjustment of pharmacological treatment, to prevent the cognitive decline of these patients and improve both their social functioning as the quality of life. In the case of younger patients, it would be essential to work on strategies to adapt to the disease, improve social skills and promote recovery to achieve a better quality of life (Andreasen, Oades \& Caputti, 2011).

The limitations of this study include the relatively small sample size, the use of a cross-sectional design, and the lack of an instrument to specifically assess negative symptoms. On the other hand, it also has certain strengths such as the global description of the sample at the level of symptoms, and cognitive and social functioning, providing us with a clear picture of the status of a sample of outpatients with chronic schizophrenia. It would be interesting for future research to carry out psychological intervention programs taking into account the age of the participants and gender; to work in the improvement of the cognitive functioning in the older subjects, and in the adaptation to the disease of the younger patients to, in this way, avoid isolation and achieve an improvement in their social rehabilitation.

Further, our study indicates areas on which we should place emphasis, especially in patients under 45 years of age, to minimise further cognitive deterioration and promote better social integration. Schizophrenia Research, 106,

Bozikas, V. P., Kosmidis, M. H., Kiosseoglou, G., \& Karavatos, A. (2006). Neuropsychological profile of cognitively impaired patients with schizophrenia. Comprehensive Psychiatry, 47, 136-143. https://doi.org/10.1016/j.comppsych.2005.05.002.

Carceller, M., Portella, M. J., Carmona, C., Rametti, G., Puigdemont, D., Figueras, M., Fernández-Vidal, A., Villalta, L., \& Álvarez, E. (2014). Efectos neuropsicológicos del tratamiento de mantenimiento con Clozapina en el trastorno psicótico resistente. Actas Españolas de Psiquiatría, $42,68-73$.

Carson, N. J. (2006). Fisiología de la conducta. Madrid: Pearson.

Cohen, C. I., Vahia, I., Reyes, P., Diwan, S., Bankole, A. O., \& Palekar, N. (2008). Focus on geriatric psychiatry: schizophrenia in later life: clinical symptoms and social well-being. Psychiatric Services, 9, 232-234. https://doi.org/10.1176/ps.2008.59.3.232. 
Comparelli, A., de Carolis, A., Emili, E., Rigucci, S., Falcone, I., Corigliano, V., Curto, M., Trovini, G., Dehning, J., Kotzalidis, G. D., \& Girardi, P. (2014). Basic symptoms and psychotic symptoms: the relationships in the at risk mental states, first episode and multi-episode schizophrenia. Comprehensive Psychiatry, 55, 785-791. https://doi.org/10.1016/j.comppsych.2014.01.006.

Corrigan, P. W., \& Watson, A. (2002). The paradox of self-stigma and mental illness. Clinical Psychology; Research \& Practice, 9, 35-53. https://doi.org/10.1093/clipsy.9.1.35.

Dickinson, D., Ramsey, M. E., \& Gold, J. M. (2007). Overlooking the obvious: a meta-analytic comparison of digit symbol coding tasks and other cognitive measures in schizophrenia. Archives of General Psychiatry, 64, 532-542. https://doi.org/10.1001/archpsyc.64.5.532.

Fernández-Martin, L. C., Fombellida, C., \& Montero-Fernández, C. (2014). Behavioral activation therapy for negative symptoms in schizophrenia: a clinical case. Norte de Salud Mental 49, 76-81.

Fervaha, G., Foussias, G., Agid, O., \& Remington, G. (2014). Motivational and neurocognitive deficits are central to the prediction of longitudinal functional outcome in schizophrenia. Acta Psychiatrica Scandinavica, 130, 290-299. https://doi.org/10.1111/acps.12289.

Foussias, G., Mann, S., Zakzanis, K. K., van Reekum, R., Agid, O., \& Remington, G. (2011). Prediction of longitudinal outcome in schizophrenia: the impact of baseline motivational deficits. Schizophrenia Research, 132, 427-439. https://doi.org/10.1016/j.schres.2011.06.026.

Friedman, J. I., Harvey, P. D., Coleman, T., Moriarty, P. J., Bowie, C. R., Parrella, M., White, L., Adler, D., \& Davies, K. L. (2001). Six-year follow-up study of cognitive and functional status across the lifespan in schizophrenia: a comparison with Alzheimer's disease and normal aging. American Journal of Psychiatry, 158, 1441-1448. http://doi.org/10.1176/appi.ajp.158.9.1441.

Fucetola, R., Seidman, L. J., Kremen, W. S., Faraone, S. V., Goldstein, J. M., \&Tsuang, M. T. (2000). Age and neuropsychologic function in schizophrenia: a decline in executive abilities beyond that observed in healthy volunteers. Biological Psychiatry, 48, 137-146. http://psycnet.apa.org/doi/10.1016/S0006-3223(00)00240-7.

Fuentes-Durá, I., Romero-Peris, M., Dasí-Vivó, C., \& Ruiz-Ruiz, J. C. (2010). Versión abreviada del WAIS III para su uso en la evaluación de pacientes con esquizofrenia. Psicothema, 22, 202-207.

Fusar-Poli, P., Borqwardt, S., Bechdolf, A., Addington, J., Riecher-Rössler, A., Schultze-Lutter, F., Keshavan, M., Wood, S., Ruhrmann, S., Seidman, L. J., Valmaggia, L., Cannon, T., Velthorst, E., de Haan, L., Cornblatt, B., Bonoldi, I., Birchwood, M., McGlashan, T., Carpenter, W., McGorry, P., Klosterkötter, J., McGuire, P., \& Young, A. (2013). The psychosis high-risk state: a comprehensive state of the art review. JAMA Psychiatry, 70,

107-120. https://doi.org/10.1001/jamapsychiatry.2013.269.

Gabaldon, O., Ruiz-Iriondo, M., \& Bobowik, M. (2013). Multi-informant perception of quality of life and adaptation in chronic schizophrenia. Spanish Journal of. Psychology, 16, 1-8.http://doi.org/10.1017/sjp.2013.98.

García-Portilla, P., \& Bobes, J. (2013). Ante el nuevo reto de identificar el síndrome negativo de la esquizofrenia. Revista de Psiquiatría y Salud Mental, 6, 141-143. http://doi.org/10.1016/j.rpsmen.2013.10.001.

Gómez-Benito, J., Guilera, G., Pino, O., Rojo, E., Tabarés-Seisdedos, R., Safont, G., Martinez-Arán, A., Franco, M., Cuesta, M. J., CrespoFacorro, B., Bernardo, M., Vieta, E., Purdon, S. E., Mesa, F., Rejas, J., and the Spanish Working Group in Cognitive Function. (2013). The screen for cognitive impairment in psychiatry: diagnostic-specific standardization in psychiatric ill patients. BMC Psychiatry, 13, 127.https://doi.org/10.1186/1471-244X-13-127.

Granholm, E., Morris, S., Asarnov, R. F., Chock, D., \& Jeste, D. V. (2000) .Accelerated age related decline in processing resources in schizophrenia: evidence from pupillary responses recorded during the span of apprehension task. Journal of the International Neuropsychological Society, 6, 3043.

Gray, J. A., \& Roth, B. L. (2007). Molecular targets for treating cognitive dysfunction in schizophrenia. Schizophrenia Bulletin, 33, 1100-1119. https://dx.doi.org/10.1093\%2Fschbul\%2Fsbm074.
Gross, G., \& Huber, G. (1996). The true onset of schizophrenia in its meaning for the view of the disorder. Neurology, Psychiatry and Brain Research, 4, 93-102.

Häfner, H., \& an der Heiden, W. (2008). Course and outcome, in: K. T. Mueser, \& D. V Jeste (Eds.), Clinical handbook of schizophrenia. New York: The Guilford Press.

Harvey, P. D., Silverman, J. M., Mohs, R. C., Parrella, M., White, L., Powchik, P., Davidson, M., \& Davis, K. L. (1999). Cognitive decline in late-life schizophrenia: a longitudinal study of geriatric chronically hospitalized patients. Biological Psychiatry, 45, 32-40.

Harvey, P. D., \& Strassing, M. (2012). Predicting the severity of everyday functional disability in people with schizophrenia: cognitive deficits, functional capacity, symptoms and health status. World Psychiatry, 11, 73-79.

Heaton, R. K., Chelune, G. J., Talley, J. L., Kay, G. G., \& Curtiss, G. (1993). Wisconsin card sorting test manual (revised an expanded). Odessa, Florida: Psychological Assessment Resources, Inc.

Heaton, R. K., Chelune, G. J., Talley, J. L., Kay, G. G., \& Curtiss, G. (2009). Test de clasificación de tarjetas de Wisconsin. Madrid: TEA Ediciones.

Jimeno-Bulnes, N., Jimeno-Valdés, A., \& Vargas-Aragón, M. L. (1996). El sindrome psicótico y el inventario de Frankefurt: conceptos y resultados. Barcelona: Springer-Verlag.

Jobe, T. H., \& Harrow, M. (2005). Long term outcome in patients with schizophrenia: a review. Canadian Journal of Psychiatry, 58, 892-900. https://doi.org/10.1177/070674370505001403.

Kirpatrick, B., Fenton, W., Carpenter, W. T., \& Marder, S. T. (2006). The NIMH-MATRICS consensus statement on negative symptoms. Schizophrenia Bulletin, 32, 296-303. https://doi.org/10.1093/schbul/sbj053.

Klosterkötter, J., Rurhman, S., Schultze-Lutter, F., Sakalongas, R. K. R., Linszen, D., Birchwood, M., Juckel, G., Morrison, A. P., VázquezBarquero, J. L., Hambrecht, M., Graf von Raventlow., and the EPOS group. (2005). The European prediction of psychosis study (EPOS): integrating early recognition and intervention in Europe. World Psychiatry, 4, 161-167.

Klosterkötter, J., Schultze-Lutter, F., Benchdorf, A., \& Rurhman, S. (2011). Prediction and prevention of schizophrenia: what has been achieved and where to go next. World Psychiatry, 10, 165-174.

Knowles, E. M., David, A. S., \& Reichenberg, A. (2010). Processing speed deficits in schizophrenia: re-examining the evidence. American Journal of $\begin{array}{lll}\text { Psychiatry, } & \text { 828-835. }\end{array}$ https://doi.org/10.1176/appi.ajp.2010.09070937.

McGrath, J., Saha, S., Welham, J., El Saadi, O., MacCauley, C., \& Chant, D. (2004). A systematic review of the incidence rate of schizophrenia: the distribution of rates and influence of sex, urbanicity, migrant status and methodology. BMC Medicine, 2, 13. https://doi.org/10.1186/17417015-2-13.

Miret, S., Fatjó-Vilas, M., Peralta, V., \& Fañanás, L. (2016). Síntomas básicos en la esquizofrenia, su estudio clínico y relevancia en investigación. Revista de Psiquiatría y Salud Mental, 9, 111-122. https://doi.org/10.1016/j.rpsm.2015.10.007.

Mueller, D. R., Schmidt, S. J., \& Roder, V. (2013). Integrated psychological therapy: effectiveness in schizophrenia inpatient settings related to patients' age. American Journal of Geriatric Psychiatry, 21, 231-241. https://doi.org/10.1016/j.jagp.2012.12.011.

NICE (National Institute for Health and Clinical Excellence) (2014). Psychosis and schizophrenia in adults: prevention and management. [CG78]. Retrieved from https://www.nice.org.uk/guidance/cg178.

Oliver, J. P. J., Huxley, P. J., Priebe, S., \& Kaiser, W. (1997). Measuring the quality of life of severely mentally ill people using the Lancashire Quality of Life Profile. Social Psychiatry and Psychiatric Epidemiology, 32, 76-83.

Orellana, G., \& Slachevsky, A. (2013). Executive functioning in schizophrenia. Frontiers in Psychiatry, 24, 2-35. https://doi.org/10.3389/fpsyt.2013.00035.

Overall, L. E., \& Gorham, D. R. (1962). The brief psychiatric rating scale. $\begin{array}{lll}\text { Psychological } & \text { Reports, } & \text { 10, }\end{array}$ http://psycnet.apa.org/doi/10.2466/pr0.1962.10.3.799.

Peralta, V., \& Cuesta, M. J. (1994). Psychometric properties of the positive and negative síndrome scale (PANSS) in schizophrenia. Psychiatry Research, 53, 31-40. 
Pino, O., Guilera, G., Gómez, J., Rojo, E. J., Vallejo, J., \& Purdon, S. E. (2006). Escala breve para evaluar el deterioro cognitivo en pacientes psiquiátricos. Psycothema, 18, 447-452.

Purdon, S. E. (2005). The Screen for Cognitive Impairment in Psychiatry (SCIP): instructions and three alternate forms. Edmonton, Alberta: PNL, Inc.

Rocca, P., Montemagni, C., Mingrone, C., Crivelli, B., Sigaudo, M., \& Bogetto, F. (2016). A cluster-analytical approach toward real-world outcome in outpatients with stable schizophrenia. European Psychiatry, 32,48-54.

Saha, S., Chant, D., Welham, J., \& McGrath, J. (2005). A systematic review of the prevalence schizophrenia. PLos Medicine, 2. https://doi.org/10.1371/journal.pmed.0020141.

Schultze-Lutter, F. (2009). Subjective symptoms of schizophrenia in research and the clinic: the basic symptoms concept. Schirophrenia Bulletin, 35, 5-8. https://dx.doi.org/10.1093\%2Fschbul\%2Fsbn139.

Schultze-Lutter, F., Ruhrmann, S., \& Klosterkötter, J. (2006). Can schizophrenia be predicted phenomenologically? In: J. O. Johannesen, B. V. Martindale \& J. Cullberg (Eds.), Evolving psychosis: different stages, different treatments. New York: Routledge.

Sullwood, L., \& Huber, G. (1986). Schizophrene Basisstörungen. Berlin: Springer.

Torres, A., \& Olivares, J. M. (2005). Validación en castellano de la Social Functioning Scale. Actas españolas de psiquiatría, 33, 216-220.
Vauth, R., Kleim, B., Wirtz, M., \& Corrigan, P. W. (2007). Self-efficacy and empowerment as outcomes of self-stigmatizing and coping in schizo$\begin{array}{llll}\text { phrenia. Psychiatry } & \text { Research, }\end{array}$ https://doi.org/10.1016/j.psychres.2006.07.005.

Vázquez-Barquero, J. L., Gaite, L., Ramírez, N., García-Usieto, E., BorraRuiz, C., Sanz-García, O., Bobes, J., \& Oliver, J. (1997). Desarrollo de la versión española del perfil de Calidad de Vida de Lancashire (LQoLP). Archivos de Neurobiología, 60, 125-139.

Weschler, D. (1999). WAIS -III. Escala de inteligencia de Wechsler para adultosIII. Madrid:TEA Ediciones.

Wils, R.S., Gotsfredsen, D. R., Hjorthoj, C., Austin, S. F., Albert, N., Secher, R. G., Elgaard-Thorup, A. A., Mors, O., \& Nordentoft, M. (2017). Antipsychotic medication and remission of psychotic symptoms 10 years after a first-episode psychosis. Schizophrenia Research, 182, $42-48$.

World Health Organization. (1992). The ICD-10 Classification of Mental and Behavioral Disorders. Clinical descriptions and diagnostic guidelines. Geneva: WHO.

World Medical Association. (2013). World Medical Association Declaration of Helsinki: ethical principles for medical research involving human subjects. JAMA, 310, 2191-2194. https://doi.org/10.1001/jama.2013.281053 